\title{
Flow cytometric assessment of activation of peripheral blood platelets in dogs with normal platelet count and asymptomatic thrombocytopenia
}

\author{
M. Żmigrodzka' ${ }^{1}$ M. Guzera², A. Winnicka' ${ }^{1}$ \\ ${ }^{1}$ Department of Pathology and Veterinary Diagnostics, Faculty of Veterinary Medicine, \\ Warsaw University of Life Sciences, ul. Nowoursynowska 159, 02-776 Warsaw, Poland \\ ${ }^{2}$ Department of Veterinary Medicine, University of Cambridge, Madingley Road, Cambridge CB3 0ES, \\ United Kingdom
}

\begin{abstract}
Platelets play a crucial role in hemostasis. Their activation has not yet been evaluated in healthy dogs with a normal and low platelet count. The aim of this study was to determine the influence of activators on platelet activation in dogs with a normal platelet count and asymptomatic thrombocytopenia. 72 clinically healthy dogs were enrolled. Patients were allocated into three groups. Group 1 consisted of 30 dogs with a normal platelet count, group 2 included 22 dogs with a platelet count between 100 and $200 \times 10^{9} / l$ and group 3 consisted of 20 dogs with a platelet count lower than $100 \times 10^{9} / l$. Platelet rich-plasma (PRP) was obtained from peripheral blood samples using tripotassium ethylenediaminetetraacetic acid (K3-EDTA) as anticoagulant. Next, platelets were stimulated using phorbol-12-myristate-13-acetate or thrombin, stabilized using procaine or left unstimulated. The expression of CD51 and CD41/CD61 was evaluated. Co-expression of CD41/CD61 and Annexin V served as a marker of platelet activation. The expression of CD41/CD61 and CD51 did not differ between the 3 groups. Thrombin-stimulated platelets had a significantly higher activity in dogs with a normal platelet count than in dogs with asymptomatic thrombocytopenia. Procaine inhibited platelet activity in all groups. In conclusion, activation of platelets of healthy dogs in vitro varied depending on the platelet count and platelet activator.
\end{abstract}

Key words: asymptomatic thrombocytopenia, canine, flow cytometry, platelet activation

\section{Introduction}

Platelet activation in a response to a blood vessel wall damage plays a crucial role in the process of hemostasis. Platelets can become activated after adhesion to extracellular matrix in the site of endothelial injury and/or in response to the action of secondary mediators such as thrombin, adenozyno-5'-difosforan (ADP) and thromboxane $\mathrm{A}_{2}$ (Boudreaux et al. 2010). The degree of platelet activation depends on the number and the density of surface receptors, composition of cytoplasmic granules and the concentration of

Correspondence to: M. Żmigrodzka, e-mail: magdalena_zmigrodzka@sggw.pl 
signaling molecules. Also other biological components such as collagen, serotonin or adrenalin, anticoagulants or phorbol esters stimulate platelet activation (Casonato et al. 1994, Moritz et al. 2003). Procaine, on the contrary, inhibits this process by stabilization of human platelet membrane (Watała et al. 1999).

Expression of integrin receptors and phosphatidylserine on the surface of platelets are considered as markers of their activation. Integrins are glycoprotein transmembrane receptors which are engaged in platelet adhesion and aggregation during the formation of a platelet plug. GP $\alpha \operatorname{IIb} \beta 3$ (CD41/CD61) and GP $\alpha$ $\beta 3$ (CD51), adhesion molecules for fibrinogen, vitronectin, thrombospondin and von Willebrand (vW) factor, are considered as one of the most important platelet antigens (Philips et al. 1988, Boudreaux et al. 2010). Human vitronectin receptor GPovß3 (CD51), on the other hand, is less abundant and is involved in the clotting cascade and in the process of fibrinolysis (Preissner and Seiffert 1998).

As the location of phosphatidylserine and other phospholipids in the platelet membrane depends on their functional status, they serve as markers of platelet activation (Dachary-Prigent et al. 1993). In non-activated platelets, phosphatidylserine is present only on the inner surface of the membrane. Platelet activation using thrombin, ADP or collagen causes its relocation to the extracellular surface through a protein kinase $\mathrm{C}$-activated phosphatidylserine translocase. Thereby, this increases platelet procoagulant activity and enhances binding of fibrinogen leading to promotion of clot formation (Bode et al. 1991, Briddon et al. 1999). However, procaine has been shown to accelerate the depolarization of the mitochondrial membrane and to activate caspase- 3 and caspase- 9 leading to an increased phosphatidylserine expression (Alberio and Dale 1999).

Asymptomatic thrombocytopenia is common in several breeds of dogs, including Cavalier King Charles Spaniel (CKCS) and Greyhound (Olsen et al. 2001, Davis et al. 2008). Mild familial thrombocytopenia was also reported in German Shepherd, Shiba Inu and Akita Inu (Dachary-Prigent et al. 1993, Steiss et al. 2000, Singh and Lamb 2005, Bertazzolo et al. 2007, Larcia-Garcia et al. 2008). Interestingly, affected individuals, despite a low platelet count, are not more prone to the development of coagulation disorders (Brown et al. 1994, Cowan et al. 2004, Bertazzolo et al. 2007, Boudreaux et al. 2010).

The aim of the study was to assess the activation of peripheral blood platelets in healthy dogs with normal platelet count and asymptomatic thrombocytopenia. Platelet phenotype was determined by the evaluation of the expression of platelet surface antigens and phosphatidylserine in K3-EDTA blood samples after incubation with platelet stabilizers, activators and without stimulation.

\section{Materials and Methods}

\section{Animals and blood samples}

Dogs with thrombocytopenia and normal platelet count were recruited in the study between January 2005 and December 2011 among patients undergoing routine blood screening at two veterinary clinics in Warsaw (Small Animal Clinic at the Faculty of Veterinary Medicine, Warsaw University of Life Sciences and Veterinary Clinic Guderskiego 5). The inclusion criteria were: lack of clinical and laboratory signs of disease, including absence of clinical coagulation disorders, no history of treatment or vaccination during the 4 weeks preceding the sampling, absence of platelet clumps in the blood smear. Dogs were assigned to 3 groups according to the platelet count in K3-EDTA-anticoagulated blood. Group 1 included dogs with a normal platelet count (between 200 and $\left.580 \times 10^{9} / l\right)$. Patients with a platelet count lower than $200 \times 10^{9} / l$ were considered thrombocytopenic and were allocated to either group 2 (dogs with platelet count between 100 and $200 \times 10^{9} / 1$ ) or group 3 (patients with platelet count lower than $\left.100 \times 10^{9} / l\right)$.

Peripheral blood samples were obtained by venipuncture from the cephalic vein into K3-EDTA tubes (Medlab, Raszyn, Poland).

\section{Hematology, clinical biochemistry and coagulation times}

Complete blood count was determined within 20 minutes after blood collection using an Abacus hematology analyzer on K3-EDTA blood samples (Diatron, Budapest, Hungary). May-Grunwald Giemsa-stained blood smears were examined under a light microscope. Serum aspartate transaminase (AST) and alanine transaminase (ALT) levels were measured with an automated biochemistry analyzer Pointe- 180 (Pointe Scientific, Warsaw, Poland) using a kinetic method. Urea concentration was evaluated on the same analyzer by urease method (indirect measurement). The absorbance was measured at $340 \mathrm{~nm}$. Prothrombin time (PT) and activated partial thromboplastin time (APTT) were assessed in citrate plasma by a K-3002 Optic Blood Coagulometer (Elza-Bis Kselmed, Grudzi dz, Poland) with nephelometric method using Dia-PT 2, Dia-APTT, Dia-CaCl2 reagents (Bio-Ksel, Grudzi dz, Poland). 


\section{Preparation of platelet rich-plasma}

Platelet-rich plasma (PRP) was obtained using K3-EDTA blood samples by centrifugation (300 g, 10 min, $20^{\circ} \mathrm{C}$ ) (Moritz et al. 2003).

\section{Identification of platelets / Identification of platelet surface receptors}

The expression of CD41/CD61 (integrin $\beta 3$ ) and CD51 ( $\alpha$ v $\beta 3$ integrin - vitronectin receptor) was evaluated using monoclonal antibodies. Platelets from K3-EDTA PRPs were suspended in a physiological phosphate buffered saline solution containing $5 \mathrm{mM}$ sodium azide, $0.1 \%$ glycine and $1 \%$ goat serum (PBS) (Watała et al. 1999, Wilkerson and Shuman 2001).

Samples were incubated for $20 \mathrm{~min}$ in the dark with a mouse anti-human CD41/CD61 antibody conjugated with fluorescein isothiocyanate (FITC) (clone VIPL2, BD Pharmingen, San Jose, USA), or a mouse anti-human CD51 antibody conjugated with phycoerythrin (PE) (clone LM609, Chemicon, Temecula, USA) (Poncz et al. 1987). Both antibodies were reported to be cross-reactive with canine antigens (Poncz et al. 1987, Schlossman et al. 1995, Lebrec et al. 2012). After washing, samples were resuspended in the buffer and analyzed.

\section{Evaluation of platelet activation}

Annexin V was used to evaluate platelet activation by detection of platelets expressing phosphatidylserine. Before staining, platelets in K3-EDTA PRP were stabilized by addition of 10 $\mathrm{mg} / \mathrm{m} l$ procaine (Polfa Tarchomin, Warsaw, Poland) and incubation for $20 \mathrm{~min}$ in the dark or activated by incubation as before but with $10 \mathrm{ng} / \mathrm{m} l$ phorbol 12-myristate 13-acetate (PMA) (Sigma-Aldrich, Saint Louis, USA) or $2 \mathrm{U} / \mathrm{m} l$ thrombin (Biomed, Warsaw, Poland) instead of procaine (Watała et al. 1999, Wilkerson and Shuman 2001, Moritz et al. 2003). After spinning $\left(220 \mathrm{~g}, 8^{\circ} \mathrm{C}\right.$ for $\left.15 \mathrm{~min}\right)$, samples were suspended in Annexin V Binding Buffer (BD Pharmingen) (Wilkerson and Shuman 2001) and incubated with Annexin V labeled with PE (BD Pharmingen) and a mouse anti-human CD41/CD61 antibody (clone VIPL2, BD Pharmingen) for $20 \mathrm{~min}$ in the dark at room temperature. Samples were then washed with buffered saline solution, spinned $(220 \mathrm{~g}$, $15 \mathrm{~min}, 8^{\circ} \mathrm{C}$ ), resuspended in the PBS and analyzed within $30 \mathrm{~min}$.

\section{Flow cytometric analysis}

Samples were analyzed using FACSCalibur flow cytometer (BD) and CellQuestPro software (BD). 10,000 events were analyzed in each sample. Platelets and their fractions were identified on the forward scatter height (FSC-H) against side scatter height (SSC-H) plot. The normoplatelet region (R1) was set to includes $>95 \%$ of the platelets. Other platelet fractions, microparticles (R2) and aggregates (R3), were defined as particles smaller or larger than normoplatelets, respectively (Fig. 1) (Moritz et al. 2003).

\section{Statistical analysis}

Results were presented as mean \pm standard deviation (SD). Statistical analysis was performed using STATISTICA 6.0 software (StatSoft, Tulsa, USA). The distribution of the data was tested by Shapiro-Wilk test. Wilcoxon signed-rank test was used to determine whether there were significant differences between procaine, PMA, thrombin or unstimulated platelets. The statistical significance of differences between patients groups was evaluated by Mann-Whitney U test. Differences were considered significant when $p<0.05$ and highly significant when $\mathrm{p}<0.01$.

\section{Results}

\section{Animals}

Seventy two dogs met the inclusion criteria and were enrolled in the study. Their age ranged from 1 year to 11 years (median 3.5 years), they were 26 intact males, 3 neutered males, 28 intact females and 15 neutered females. Among thirty dogs included in group 1, sixteen were of mixed-breed, five were Greyhounds, three Standard Schnauzers, three Miniature Schnauzers, two Giant Schnauzers and one was German Shepherd. The age ranged from 1 year to 11 years (median 4 years), they were 8 intact males, 2 neutered males, 8 intact females and 12 neutered females. Group 2 consisted of twenty two dogs, of which fifteen were Cavalier King Charles Spaniels, four Greyhounds, two German Shepherds and one was Miniature Schnauzer. They were 1 year to 9 years old (median 3.5 years) and 10 intact males, 10 intact females and 2 neutered females. Group 3 included twenty dogs, of which seventeen were Cavalier King Charles Spaniels and three were Greyhounds. Their age ranged from 1 year to 8 years old (median 3.5) and they were 8 intact males, 1 neutered male, 10 intact females and 1 neutered female. 


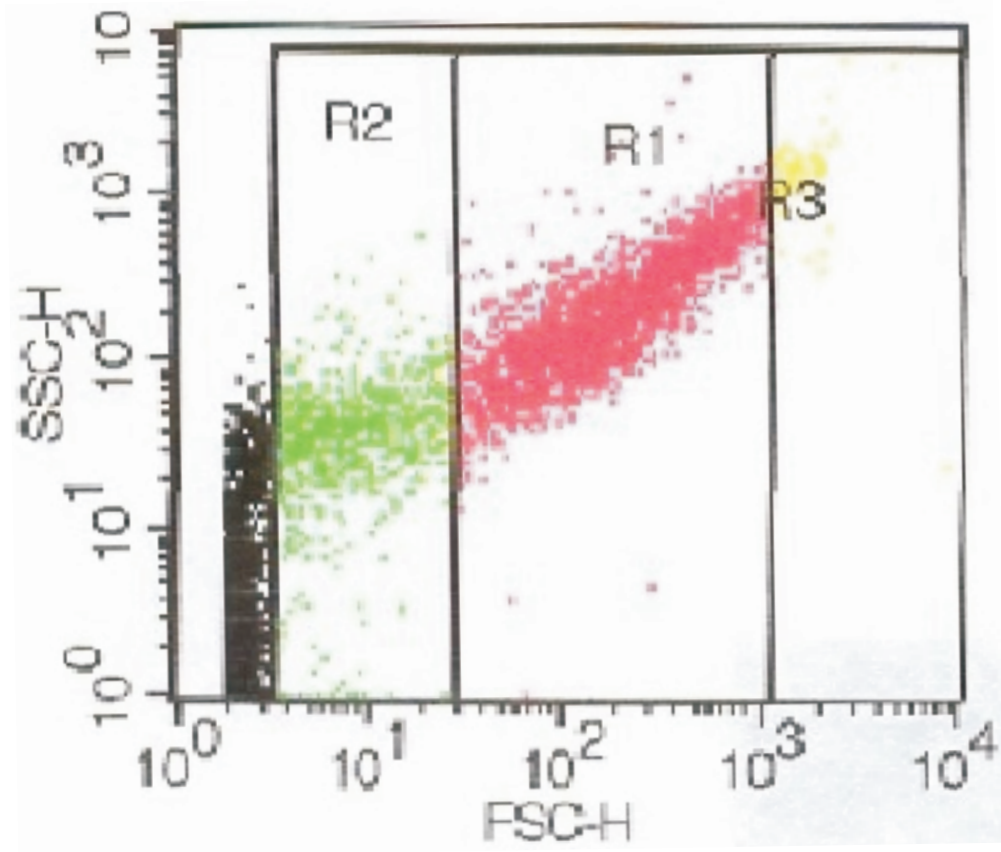

Fig. 1. Representative forward scatter height (FSC-H) versus side scatter height (SSC-H) plots showing the gating strategy of platelet fractions: normoplatelets (R1), platelet microparticles (R2), and aggregates (R3) in tripotassium ethylenediaminetetraacetic acid (K3-EDTA) platelet-rich plasma (PRP).

\section{PRP K3-EDTA}

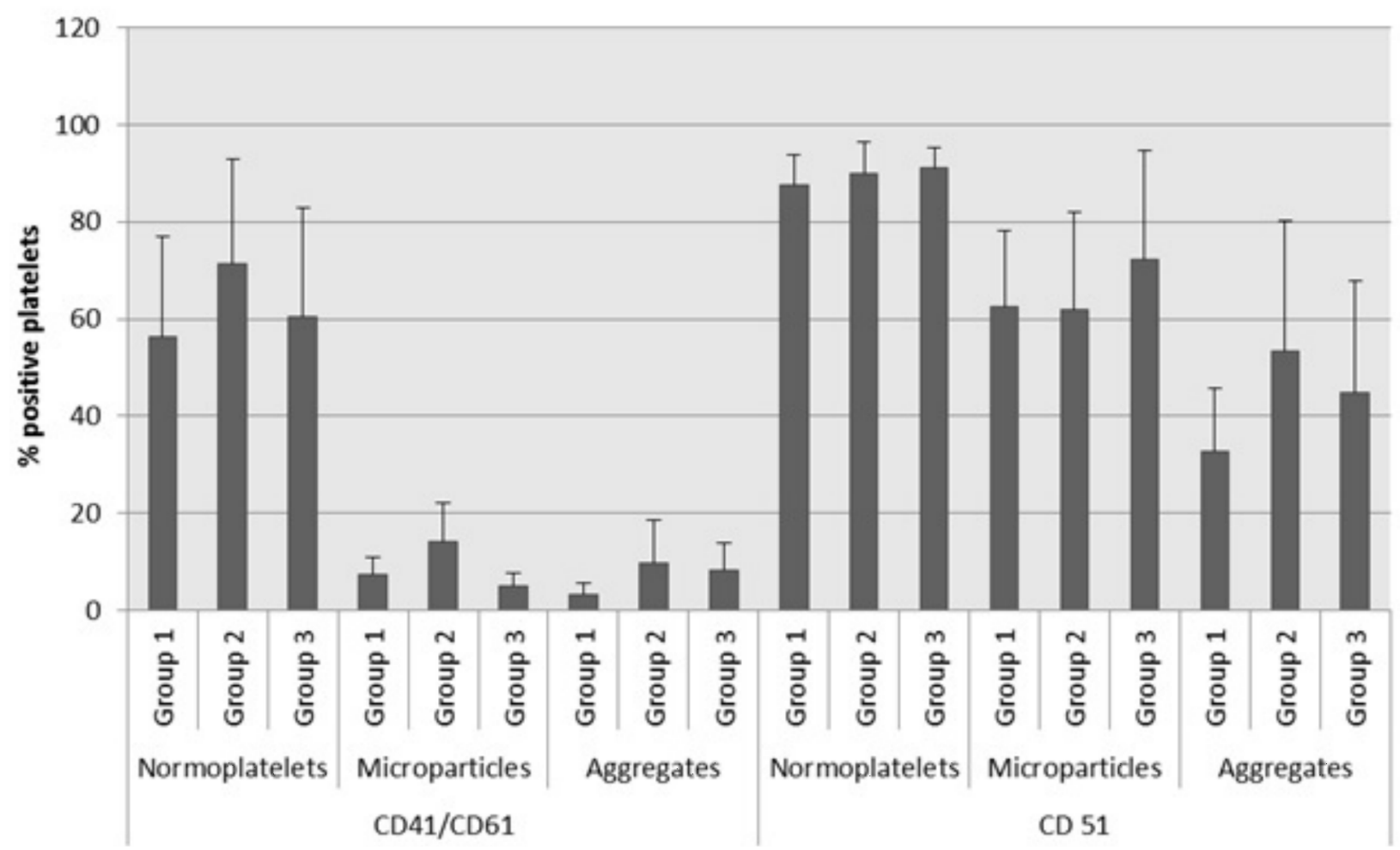

Fig. 2. The percentage of CD41/CD61+ and CD51+ normoplatelets, platelet microparticles, and aggregates in tripotassium ethylenediaminetetraacetic acid (K3-EDTA) platelet-rich plasma (PRP) in dogs with a platelet count between 200 and $580 \times 10^{9} / 1$ (group 1) $(\mathrm{n}=30)$, between 100 and 200x10 $/ 1$ (group 2) $(\mathrm{n}=22)$, and below $100 \times 10^{9} / 1$ (group 3) $(\mathrm{n}=20)$. Mean \pm standard deviation $(\mathrm{SD})$. 


\section{Clinical and clinicopathological findings}

Patients' history, physical examination, hematology, clinical chemistry and coagulation times were unremarkable. However, some dogs had asymptomatic thrombocytopenia and they were included in group 2 or group 3. None of the patients had platelet clumps present in the blood smears.

\section{Expression of platelet surface receptors}

It was shown that the percentage of CD51 and CD41/CD61+ platelets in the normoplatelet and microparticles fraction in all 3 groups was not statistically significant in comparison to unstimulated sample (Fig. 2).

\section{Platelet activation}

Percentage of platelets co-expressing CD41/CD61 and Annexin V in the K3-EDTA PRP with or without stimulation is presented in Fig. 3.

In dogs with a normal platelet count (group 1) the K3-EDTA PRP incubated with procaine had a significantly lower percentage of normoplatelets and platelet microparticles co-expressing CD41/CD61 and Annexin $\mathrm{V}$ than in unstimulated samples. The numbers of double-positive normoplatelets and microplatelets were highly significantly lower in comparison with the unstimulated samples in dogs with thrombocytopenia (group 2 and 3). In dogs with a normal platelet count and with thrombocytopenia the expression of CD41/CD61 in aggregates in PRP incubated with procaine was not statistically significantly different in comparison to unstimulated sample.

The numbers of double-positive normoplatelets after PMA activation in PRP were highly significantly greater in comparison with the unstimulated samples in normal dogs, but in microplatelets and aggregates regions there were no statistically significant differences. In dogs with a platelet count between 100 and $200 \times 10^{9} / l$ (group 2) the numbers of CD41/CD61+ Annexin $\mathrm{V}+$ platelets were highly significantly lower in comparison with the control samples only in aggregates region in PMA samples.

In group 3 (dogs with platelet count below $\left.100 \times 10^{9} / l\right)$ the co-expression of CD41/CD61 and Annexin $\mathrm{V}$ in normoplatelet and aggregate subpopulation in the K3-EDTA samples was highly significantly lower after incubation with PMA in comparison to unstimulated sample.

The percentage of normoplatelets, platelet microparticles and platelets aggregates was significantly lower or highly significantly lower after activation with thrombin in comparison with the unstimulated samples in all dogs (group 1, 2 and 3).

\section{Discussion}

Our study confirmed that the expression of CD51 and CD41/61 on platelets surface does not vary between platelets regions and between dogs with normal platelets count and dogs with thrombocytopenia. Lack of differences in the expression of vitronectin and integrin $\alpha_{\mathrm{IIb}} \beta_{3}$ receptors between groups suggests that their conformation is stable in the K3-EDTA samples.

The results of this study have also confirmed that procaine is an inhibitor of platelet activity in both, dogs with a normal platelet count and dogs with thrombocytopenia. The percentage of CD41/CD61+ Annexin $\mathrm{V}+$ platelets was lower in the presence of K3-EDTA in all platelet regions in comparison with unstimulated samples.

The anticoagulant properties of K3-EDTA are a result of removal of ions from the platelet environment. Elimination of calcium and magnesium decreases the stability of certain platelet membrane glycoproteins, thus causing transmission of the signal into the cells and initiation of release of alpha-granules. An increase in intracellular concentration of $\mathrm{Ca}^{2+}$ is one of the factors responsible for platelet microparticles formation (Blockmans et al. 1995, Boudreaux et al. 2010). The percentage of microplatelets stabilized by procaine was lower in comparison with unstimulated samples in all dogs groups and highly significantly lower in groups 2 and 3, but in group 1 it was significantly lower. The above finding confirms that procaine is a weak platelet membrane stabilizer. Procaine has been proven to selectively inhibit platelet activation in samples anticoagulated with acid citrate dextrose. Depending on its concentration it stabilizes platelet cell membrane, inhibits the release of calcium and decreases mobilization of calcium from the delta-granules (Watała et al. 1999).

PMA, a protein kinase $\mathrm{C}$ stimulator, slowly activates platelets causing their degranulation but not aggregation (Zucker and Nachmins 1985, Moritz et al. 2003). Moritz et al. (2003) have shown that a higher percentage of activated canine platelet microparticles and aggregates is present in the citrate blood after activation with PMA in comparison with the K3-EDTA PRP, by evaluation of selectin P expression. Ex vivo stimulation with PMA caused a marked increase in CD62P expression in normoplatelets and a mild increase in the region of platelet microparticles and aggregates (Moritz et al. 2003). 


\section{PRP K3-EDTA}

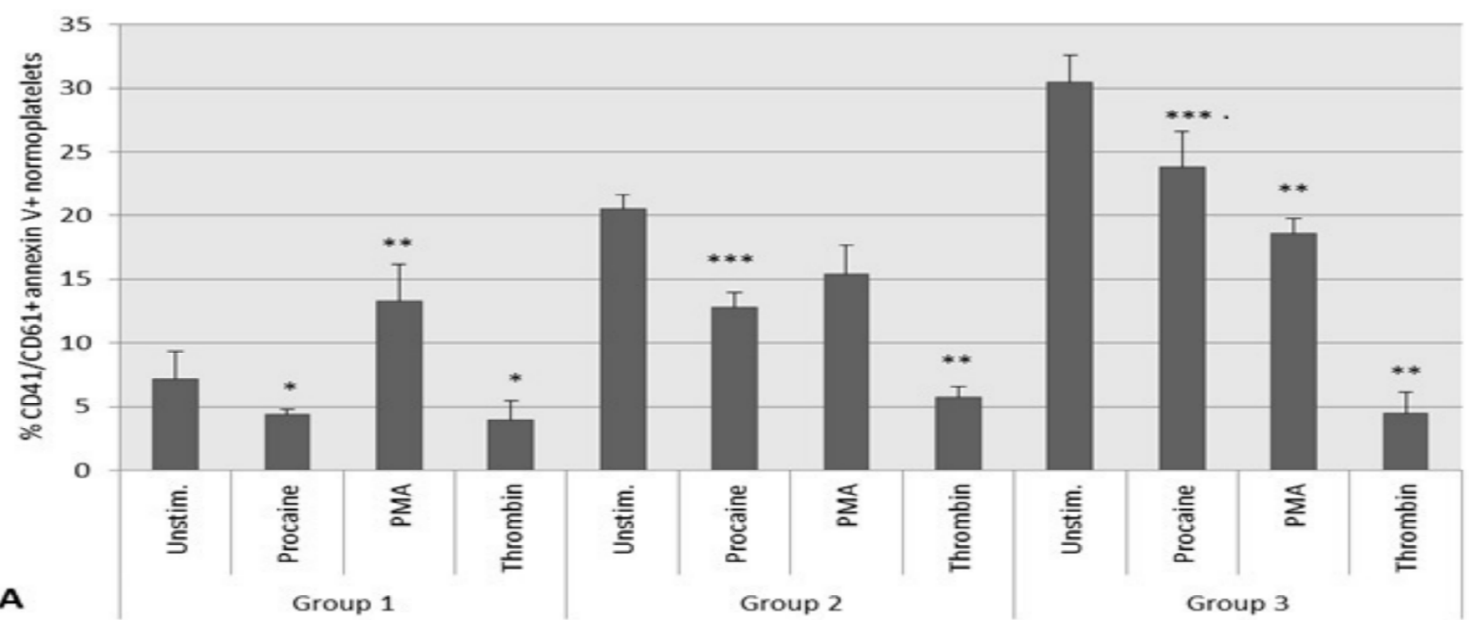

PRP K3-EDTA

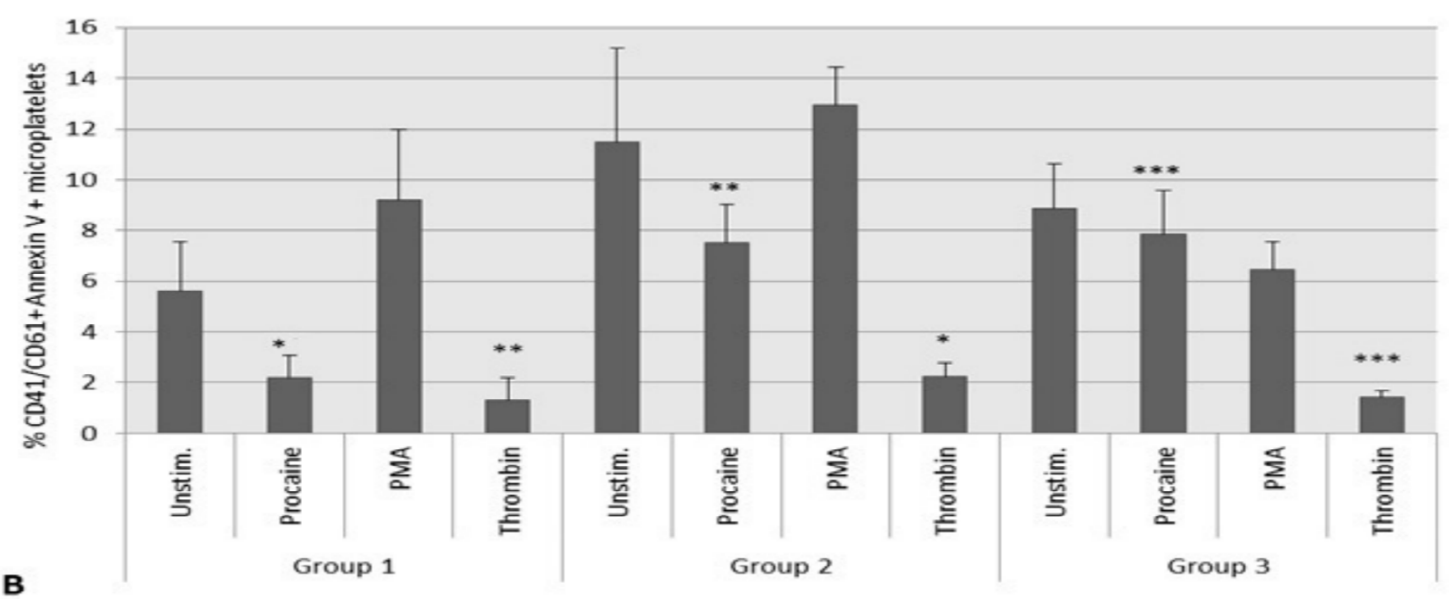

PRP K3-EDTA

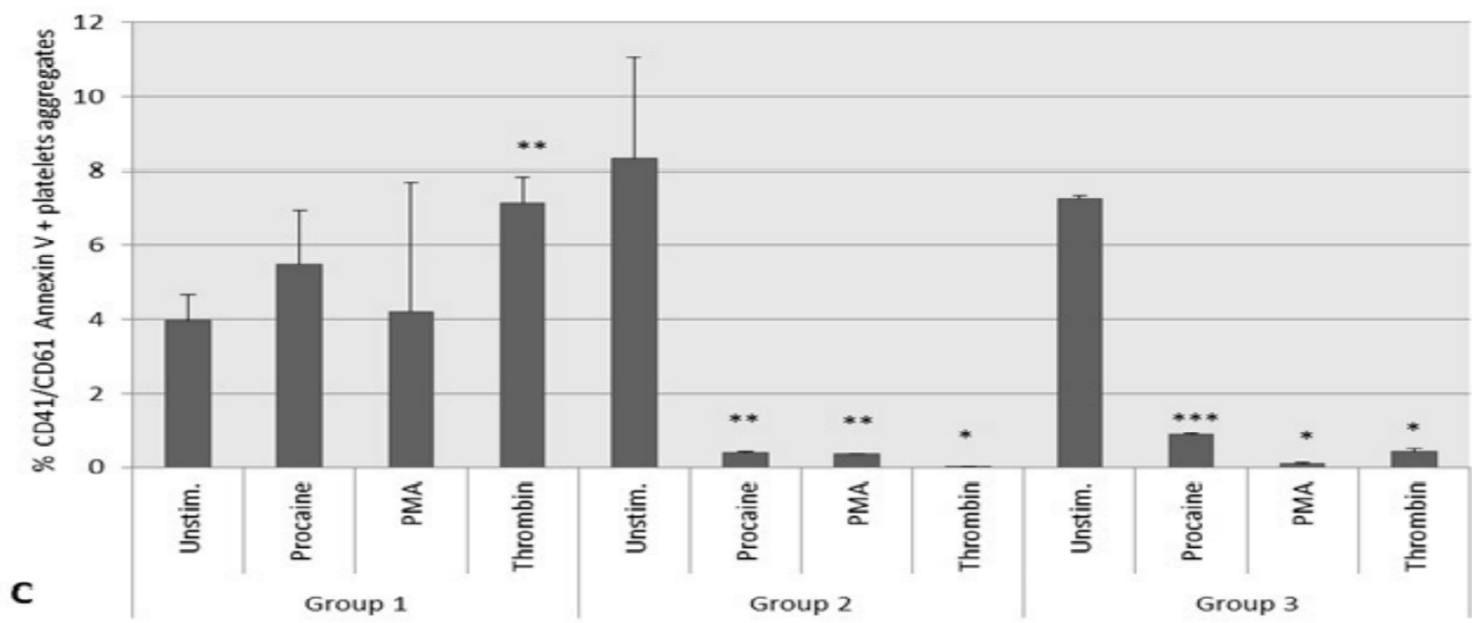

Fig. 3. The percentage of CD41/CD61+ annexin V+ normoplatelets (A), platelet microparticles $(\mathbf{B})$, and aggregates $(\mathbf{C})$ in tripotassium ethylenediaminetetraacetic acid (K3-EDTA) platelet-rich plasma (PRP) after stimulation with procaine, phorbol 12-myristate 13-acetate (PMA) or thrombin or unstimulated in dogs with a platelet count between 200 and 580x10 $/ 1$ (group 1) $(\mathrm{n}=30)$, between 100 and 200x10/1 (group 2) $(\mathrm{n}=22)$, and below 100x10 $/ 1$ (group 3) $(\mathrm{n}=20)$. Mean \pm standard deviation $(\mathrm{SD})$. Significant differences in comparison to unstimulated platelets ${ }^{*} \mathrm{p}<0.05,{ }^{* *} \mathrm{p}<0.01,{ }^{* * *} \mathrm{p}<0.001$. 
Our study confirmed that PMA is a platelet activator in dogs with a normal platelet count. PMA activation is less prominent in dogs with thrombocytopenia. In addition, the percentage of activated normoplatelets $(\mathrm{CD} 41 / \mathrm{CD} 61+$ Annexin V+) in the K3-EDTA samples in dogs with a normal platelet count was higher than in the unstimulated samples. The percentage of activated platelets in group 2 was lower in the region of platelet aggregates, whereas, in group 3 it was lower in the region of normoplatelets and aggregates. This findings are in agreement with observation made by Moritz et al. (2003) and confirm the previous observation suggesting that PMA is a slow platelet activator (Whiss et al. 1998, Moritz et al. 2003).

GP $\alpha \operatorname{IIb} \beta 3$ (CD41/CD61) is the most abundant of all platelet receptors. It is present not only on the cell surface but also in platelet alpha and delta granules and in the open canalicular system. Degranulation of platelet alpha-granules causes externalization of this receptor leading to its increased expression on the cell surface, making it an early marker of platelet activation (Poncz et al. 1987, Blockmans et al. 1995). Activation of human platelets by thrombin or collagen causes changes in the conformation of CD41/CD61 through phosphorylation of cytoskeletal and cytoplasmic proteins, allowing binding of fibrinogen and other cell adhesion ligands in the process of platelet aggregation (Philips et al. 1988, Blockmans et al. 1995, Boudreaux et al. 2010). It has been also shown that its expression is reduced in the later phases of clot formation due to its decreased accessibility to antibodies after binding of fibrinogen (Philips et al. 1988). As shown by this study the percentage of activated platelet aggregates after stimulation with thrombin was higher in dogs with a normal platelet count in comparison with unstimulated cells what confirms that thrombin is a strong physiologic platelet activator. A similar finding was not identified in group 2 or group 3 suggesting that platelets from thrombocytopenic dogs are less prone to thrombin activation. This observation may be explained by a weaker enzymatic action of thrombin on the protease-activated receptor-1 (PAR-1) and protease-activated receptor-4 (PAR-4) in comparison with dogs with a normal platelet count.

A limitation of this study was a risk of obtaining an artifactual platelet count due to the limited accuracy of the currently available diagnostic techniques. It could have led to misallocation of patients into the 3 groups. Since the dogs which had platelet clumps present in the blood smear were not included in the study, the likelihood of incorrect classification should not be considered as high. This eliminates the risk of EDTA-induced thrombocytopenia and further decreases the danger of enrolling in the study dogs with pseudothrombocytopenia.

In contrast to the protocol of PRP preparation suggested by Moritz et al. (2003), physiological phosphate buffered saline solution used in this study did not contain formaldehyde (Moritz et al. 2003). Authors decided not to use formaldehyde following the literature data suggesting that it may influence the cell size and CD41/CD61 receptor properties (Watała et al. 1999, Wilkerson and Shuman 2001).

Interestingly, unlike in nucleated cells, phosphatidylserine expression on the surface of peripheral blood platelets indicates their activation and has not been associated with the presence of any metabolic pathways characteristic for apoptosis (Bode et al. 1991, Dachary-Prigent et al. 1993).

Further research should assess the platelet activation in a greater number of dogs, including CKCS, as CKCS have been shown to be predisposed to a mitral valve insufficiency. Nearly all CKCS are affected. Dogs of this breed are a good candidate for an animal model of the human equivalent of this disease (Beadrow and Buchanan 1993). A greater availability of platelet activation markers such as $\mathrm{CP} 62 \mathrm{P}$ (indicator of platelet degranulation) and PAC-1 (active fibrinogen receptor), could allow a more in-depth assessment of the course of platelet activation. Further studies of platelet functions should also focus on microplatelets, a particles with proven proinflammatory properties which participate in atherosclerotic plaque formation.

Peripheral blood platelets are an interesting object of research which extends beyond the field of hemostasis. Platelets are involved in a number of pathological processes. Studies evaluating the mechanisms of platelet activation and identifying factors which affect them are important problem of research in medicine. Further studies should also clarify the relationship between platelets and mitral valve insufficiency in CKCS. Studies in this field should be considered particularly important considering findings of Idriss et al. (2014) who demonstrated the role of platelets and microplatelets in the development of the mitral valve insufficiency in humans (Idriss et al. 2014).

In conclusion, the platelet activation was the strongest in dogs with a normal platelet count. Thrombin-stimulated platelets had a higher activity in dogs with a normal platelet count than in dogs with thrombocytopenia. Procaine decreased the number of activated platelets. The expression of CD51 and CD41/CD61 did not differ between the dogs with a normal and low platelet count. 


\section{Acknowledgments}

This study was supported by the: European Social Fund and the public funds under The Integrated Regional Operational Programme 2004-2006 Project - Mazovian Doctoral Scholarship 2008/2009 no $747 / 1068 / 09$. Study co-financed by the State Committee for Scientific Research, grant No. 1929/B/P01/2008/35.

\section{References}

Alberio L, Dale GL (1999) Platelet-collagen interactions: membrane receptors and intracellular signalling pathways. Eur J Clin Invest 29: 1066-1076.

Beardow AW, Buchanan JW (1993) Chronic mitral valve disease in cavalier King Charles spaniels: 95 cases (1987-1991). J Am Vet Med Assoc 203: 1023-1029.

Bertazzolo W, Corazzi S, Sesso L, Scarpa P, Ru G, Paltrinieri S (2007) Comparison of methods for determining platelet numbers and volume in Cavalier King Charles spaniels. J Small Anim Pract 48: 556-561.

Blockmans D, Deckmyn H, Vermylen J (1995) Platelet activation. Blood Rev 9: 143- 156.

Bode AP, Orton SM, Frye MJ, Udis BJ (1991) Vesiculation of platelets during in vitro aging. Blood 77: 877-895.

Boudreaux MK, Weiss DJ, Wardrop KJ, Brooks MB, Callan MB, Messick JB, Modiano JF, Moritz A, Raskin RE, Teske E, Wellman ML (2010) Platelets in Schalm's Veterinary Hematology. Lippincott Williams and Wilkins 2-476.

Briddon SJ, Melford SK, Turner M, Tubylewicz V, Waston SP (1999) Collagen mediates changes in intracellular calcium in primary mouse megakaryocytes through sykdependent and - independent pathways. Blood 93: 3847-3855.

Brown SJ, Simpson KW, Baker S, Spagnoletti MA, Elwood CM (1994) Macrothrombocytosis in cavalier King Charles spaniels. Vet Rec 135: 281-283.

Casonato A, Bertomoro A, Pontara E, Dannhauser D, Lazzaro AR, Girolami A (1994) EDTA dependent pseudothrombocytopenia caused by antibodies against the cytoadhesive receptor of platelet gpIIB-IIIA. J Clin Pathol 47: 625-630.

Cowan SM, Bartges JW, Gompf RE, Hayes JR, Moyers TD, Snider CC, Gerard DA, Craft RM, Muenchen RA, Carroll RC (2004) Giant platelet disorder in the Cavalier King Charles Spaniel. Exp Hematol 32: 344-350.

Dachary-Prigent J, Freyssinet JM, Pasquet JM, Carron JC, Nurden AT (1993) Annexin V as a probe of aminophospholipid exposure and platelet membrane vesiculation: a flow cytometry study showing a role for free sulfhydryl groups. Blood 10: 2554-2565.
Davis B, Toivio-Kinnucan M, Schuller S, Boudreaux MK (2008) Mutation in beta1-tubulin correlates with macrothrombocytopenia in Cavalier King Charles Spaniels. J Vet Intern Med 22: 540-545.

Idriss NK, Blann AD, Sayed DM, Gaber MA, Hassen HA, Kish YT (2014) Circulating Endothelial Cells and Platelet Microparticles in Mitral Valve Disease With and Without Atrial Fibrillation. Angiology 66: 631-637.

Lara-Garcia A, Couto CG, Iazbik MC, Brooks MB (2008) Postoperative Bleeding in Retired Racing Greyhounds. J Vet Intern Med 22: 525-533.

Lebrec H, O'Lone R, Freebern W, Komocsar W, Moore $P$ (2012) Survey: immune function and immunotoxicity assessment in dogs. J Immunotoxicol 9: 1-14.

Moritz A, Walcheck BK, Weiss DJ (2003) Flow cytometric detection of activated platelets in the dog. Vet Clin Pathol 32: 6-12.

Olsen LH, Kristensen AT, Haggström J, Jensen AL, Klitgaard B, Hansson H, Pedrsen HD (2001) Increased platelet aggregation response in Cavalier King Charles Spaniels with mitral valve prolapse. J Vet Intern Med 15: 209-216.

Philips DR, Charo IF, Parise LV, Fitzgerald LA (1988) The platelet membrane glycoprotein IIb-IIIa complex. Blood 71: 831-843.

Poncz M, Eisman R, Heidenreich R, Silver SM, Vilaire G, Surrey S, Schwartz E, Bennett JS (1987) Structure of the platelet membrane glycoprotein IIb. Homology to the alpha subunits of the vitronectin and fibronectin membrane receptors. J Biol Chem 262: 8476-8482.

Preissner KT, Seiffert D (1998) Role of vitronectin and its receptors in haemostasis and vascular remodeling. Thromb Res 89: 1-21.

Schlossman SF, Boumsell L, Gilks W, Harlan JM, Kishimoto T, Morimoto C, Ritz J, Shaw S, Silverstein R, Springer $\mathrm{T}$ (1995) Leukocyte Typing V. New York: Oxford University Press, pp 283-290.

Singh MK, Lamb WA (2005) Idiopathic thrombocytopenia in Cavalier King Charles Spaniels. Aust Vet J 83: 700-703.

Steiss J, Brewer WG, Welles E, Wright J (2000) Hematologic and serum biochemical reference values in retired Greyhounds. Compend Cont Educ Pract Vet 22: 243-248.

Watała C, Boncler M, Golanski J, Koziołkiewicz W, Walkowiak B, Cierniewski CS (1999) Release of Calcium and P-Selectin from Intraplatelet Granules Is Hampered by Procaine. Thromb Res 94: 1-11.

Whiss PA, Andersson RG, Srinivas U (1998) Kinetics of platelet P-selectin mobilization: concurrent surface expression and release induced by thrombin or PMA, and inhibition by the NO donor SNAP. Cell Adhes Commun 6: 289-300.

Wilkerson MJ, Shuman W (2001) Alterations in normal canine platelets during storage in EDTA anticoagulated Blood. Vet Clin Pathol 30: 107-113.

Zucker MB, Nachmias VT (1985) Platelet activation. Arteriosclerosis 5: 2-18. 